\title{
Comparative expressed sequence hybridization detects recurrent patterns of altered sequence expression in oral squamous cell carcinoma
}

\author{
SNJEZANA JANJETOVIC ${ }^{1,3}$, CARSTEN STICHT ${ }^{1,4}$, KARL KNOEPFLE $^{1,5}$, STEFAN JOOS ${ }^{1,6}$, \\ CHRISTOF HOFELE $^{2}$, PETER LICHTER ${ }^{1}$ and KOLJA FREIER ${ }^{2}$ \\ ${ }^{1}$ Abteilung Molekulare Genetik (B060), Deutsches Krebsforschungszentrum; ${ }^{2}$ Klinik für Mund-Kiefer-Gesichtschirurgie, \\ Universitätsklinikum Heidelberg, Im Neuenheimer Feld 400, D-69120 Heidelberg, Germany
}

Received February 24, 2010; Accepted April 28, 2010

DOI: $10.3892 /$ or_00000869

\begin{abstract}
Despite its common histology and presentation, oral squamous cell carcinoma (OSCC) is associated with widely varying clinical behaviour and response to therapy. To further elucidate the molecular basis of OSCC, an approach for gene expression analysis termed comparative expressed sequence hybridization (CESH) was used in the present study. This straightforward approach allows the rapid delineation of pathophysiologically interesting candidate chromosome regions by a direct detection of aberrant transcriptional activation. CESH profiling of OSCC specimens led to the identification of several novel chromosomal regions. Increased expression compared to a set of control mucosa specimens was found on 1q22-q23, 3q26.3-qter, 4q31.1-q32, 11q12$\mathrm{q} 13.2,14 \mathrm{q} 32,18 \mathrm{q} 12,19 \mathrm{q} 13.2-\mathrm{q} 13.3$ and 22q13.1-q13.2. Decreased expression was found on 8p22-p23, 16p12 and 16q23-q24. Using CESH, common patterns of altered sequence expression in different OSCC samples were obtained. While some of these regions overlap with those known to be frequently altered in OSCC on the genomic level, this screen revealed novel chromosome subregions with increased trans-
\end{abstract}

Correspondence to: Dr Kolja Freier, Klinik für Mund-KieferGesichtschirurgie, Universitätsklinikum Heidelberg, Im Neuenheimer Feld 400, D-69120 Heidelberg, Germany

E-mail: kolja.freier@med.uni-heidelberg.de

Present addresses: ${ }^{3}$ II. Medizinische Klinik und Poliklinik, Universitätsklinikum Hamburg-Eppendorf, Martinistraße 52, D20246 Hamburg; ${ }^{4}$ Zentrum Medizinische Forschung, Medizinische Fakultät Mannheim, Theodor-Kutzer-Ufer 1-3, D-68167 Mannheim; ${ }^{5}$ Paul-Ehrlich-Institut Bundesamt für Sera und Impfstoffe, PaulEhrlich-Straße 51-59, D-63225 Langen; ${ }^{6}$ Helmholtz Association of German Research Centres, Anna-Louisa-Karsch-Str. 2, D-10178 Berlin, Germany

Key words: oral squamous cell carcinoma, comparative expressed sequence hybridization, mRNA criptional activity, which are probably independent of the genomic status of the tumor cells.

\section{Introduction}

Oral squamous cell carcinoma (OSCC) is the 10th most common human malignancy worldwide affecting more than 500000 individuals per year. The 5-year survival rate for oral squamous cell carcinoma does not currently exceed $55 \%$, which is mainly caused by locally aggressive tumor phenotypes and early loco-regional metastases (1). In general, clinicopathological parameters such as the TNM system, which are used as basis for therapeutic decisions, do not adequately predict the biological behaviour of the tumors. To improve the clinical management of individual patients, there is a strong requirement for a better understanding of molecular events involved in OSCC formation. Genetic changes associated with malignant transformation and tumor progression critically affect the expression of key genes. To further elucidate the molecular basis of these critical events, an approach termed comparative expressed sequence hybridization $(\mathrm{CESH})$ was recently introduced in molecular analyses of tumor specimen (2). The principles of CESH are similar to chromosomal comparative genomic hybridization (cCGH), but instead of differently labeled genomic DNA, cDNA sequences derived from cellular RNA are used as hybridization probe for test and control specimen. This allows the analysis of global expression patterns of both coding and non-coding genes in tumor specimens. Since its first publication, the CESH method was established and applied on different subtypes of rhabdomyosarcoma (RMS), Wilms' tumors, hairy cell leukemia, acute lymphoblastic leukemia and breast cancer (3-5). It was shown that individual tumor subtypes within these tumor entities could be distinguished by their expression profiles obtained by CESH. Moreover, combining cCGH and $\mathrm{CESH}$ experiments allowed the correlation of chromosome copy number changes and transcriptional activities within the same chromosome subregions $(3,6)$ further narrowing the alterations, which might cause effects in tumor development. In this study we applied CESH to a series of 28 cases of advanced OSCC in order to obtain an overview of global gene expression. Comparing the CESH data to existing cCGH data, 

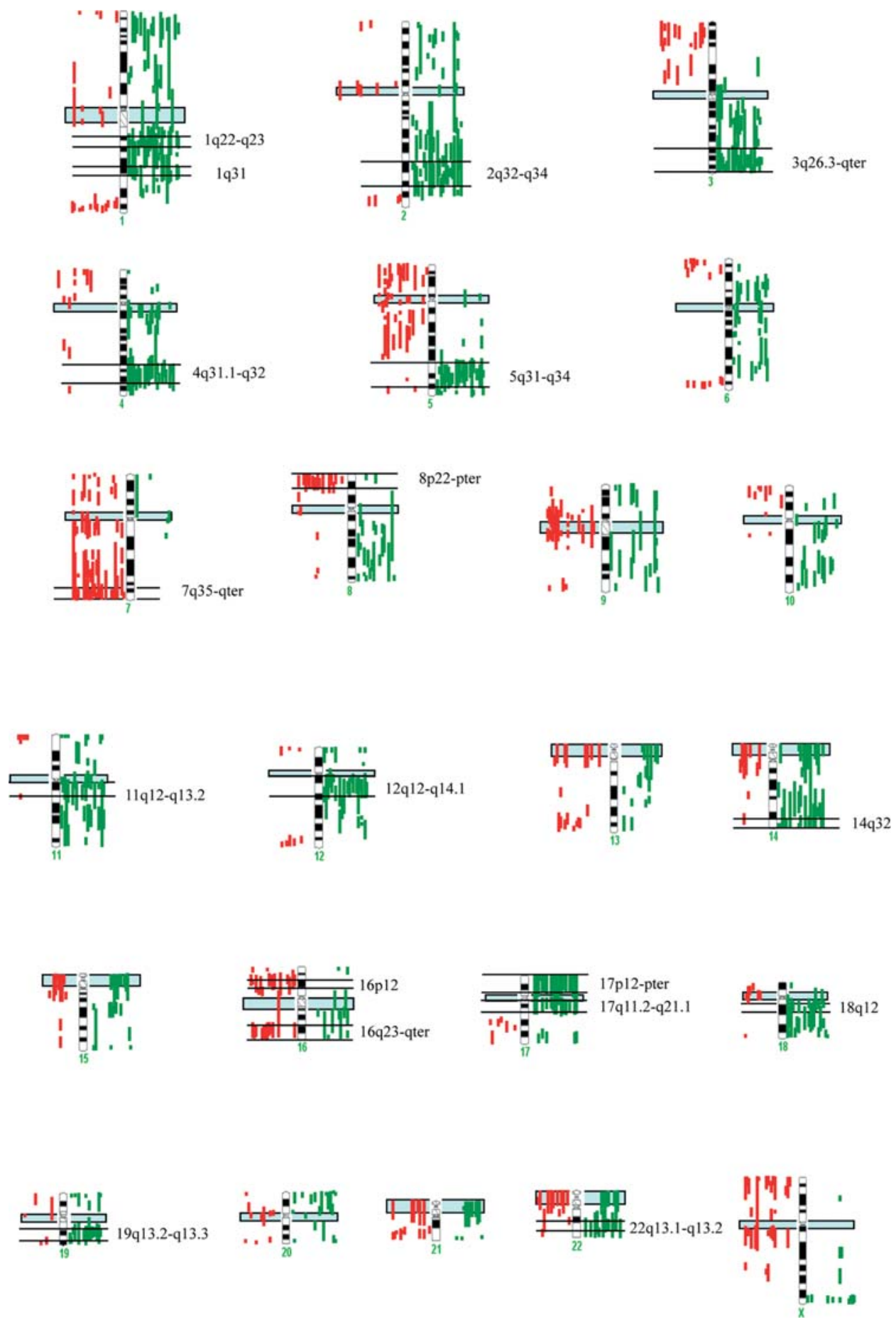

Figure 1. Schematic overview of changes in relative mRNA expression in 28 cases of OSCC detected by CESH. Red and green bars on the left and right side of chromosomal ideograms indicate relative low or high average expression levels, respectively. Blue boxes represent centromeric regions of the chromosomes. The chromosome regions most commonly altered in expression are indicated by the horizontal lines.

we aimed to identify the relationship between copy number changes and expression profile at the corresponding chromosome regions.

\section{Materials and methods}

Tumor material. Twenty-eight frozen-tissue tumor samples were collected from patients with histological confirmed OSCC after approval by the institutional review board of the
University Hospital Heidelberg and informed consent. As control tissue, oral mucosa from 4 healthy donors was used. As reference, human reference RNA from a commercially available pool of ten different cell lines was used (Stratagene, Amsterdam, The Netherlands).

RNA preparation and reverse transcription. DNA and RNA extraction from OSCC samples was carried out by ultracentrifugation in cesium chloride as previously described 
$(7,8)$. RNA quality and concentration were tested with the Agilent 2100 bioanalyzer (Agilent Technologies, Palo Alto, USA). RNA was reversely transcribed using random hexamer primers and Superscript II (Invitrogen, Karlsruhe, Germany), according to the manufacturer's instructions.

Complementary DNA labeling. Complementary DNAs $(100 \mathrm{ng})$ reverse transcribed from tumor, control and reference RNA were differently labeled with Cy3-dCTP (Amershime Biosciences) and biotin-dCTP (Loxo-Life Sciences) using BioPrime DNA Labeling Kit (Invitrogen). Unincorporated nucleotides, random primers and dyes were removed by use of the QIAquick PCR Purification kit (Qiagen, Hilden) according to the manufacturer's instructions. Incorporation of dyes into the labeled DNA was determined by UV spectrophotometry.

Hybridization to metaphase chromosomes. Five micrograms of each differently labeled probes (tumor vs. reference, and control vs. reference) were denatured together with $5 \mu \mathrm{g}$ of unlabeled Cot-1 DNA in $5 \mu 1$ of deionized formamide and $5 \mu 1$ of hybridization mixture (20\% dextransulphate/4X SSC) at $75^{\circ} \mathrm{C}$ for $7 \mathrm{~min}$ followed by pre-annealing at $37^{\circ} \mathrm{C}$ for $20 \mathrm{~min}$. Human metaphase slides (Abbott, Wiesbaden, Germany) were pretreated with $70 \%$ acetic acid for $10 \mathrm{~min}$, washed with PBS, treated with RNase A for $30 \mathrm{~min}(0.1 \mu \mathrm{g} / \mathrm{ml}$, diluted in PBS) in a humidified chamber at $37^{\circ} \mathrm{C}$ and rinsed in $2 \mathrm{X}$ SSC. After dehydration, denaturation step was performed in $70 \%$ formamide (49 $\mathrm{ml}$ formamide, $7 \mathrm{ml} \mathrm{2X} \mathrm{SSC,} 14 \mathrm{ml} 0.5 \mathrm{M}$ $\mathrm{NaPO}_{4}$ ) for $2 \mathrm{~min}$ at $70^{\circ} \mathrm{C}$. After dehydration $(70,90$ and $100 \%$ ethanol), $10 \mu 1$ of hybridization mixture with denatured probe was applied to the denatured chromosomes on the slide. Hybridization was carried out in a humified chamber at $37^{\circ} \mathrm{C}$ for $72 \mathrm{~h}$. After hybridization, slides were washed in $50 \%$ formamide, 20X SSC (pH 7.0) and 0.5X SSC (pH 7.0) at $42^{\circ} \mathrm{C}$. Incubation with avidin-FITC antibody $(2 \mu \mathrm{g} / \mathrm{ml})$ was performed for $30 \mathrm{~min}$ at $37^{\circ} \mathrm{C}$ in humified chamber, followed by incubation with $2 \mu \mathrm{g}$ of anti-avidin-FITC antibody (VectorBurlingame, Canada). Finally, the slides were washed with $4 \mathrm{X}$ SSC, $1 \%$ Tween $(\mathrm{pH} 7.3)$ at $42^{\circ} \mathrm{C}$, and mounted in an antifade solution containing 4',6-diamidino-2-phenylindole to visualize cell nuclei.

Image acquisition and the analysis. Images were acquired through a Zeiss Axioplan fluorescence microscope equipped with filter sets appropriate for DAPI, FITC and Cy3 by using CCD camera. For quantitative analysis of CESH experiments, commercially available analysis software, CytoVision 3.6 (Applied Imaging, Thunderland, UK) was used. For each metaphase spread, three digital images were acquired, one image for each fluorochrome. At least 15 metaphase spreads were analyzed for each tumor sample examined.

Definition of cut-off values for CESH experiments using standard reference intervals (SRIs). As thresholds for determination of a relative increase or decrease of expression, dynamic standard reference intervals (SRIs) were used (5). SRIs were created in order to reduce the amount of false positive and false negative results and to detect the relative expression profile by CESH with higher specificity and sensitivity (9-11). They were generated by the analysis of 100 metaphase spreads after hybridization of the same but differently labelled cDNA. The confidence intervals of $95 \%$ were used in this analysis.

\section{Results}

CESH profile of oral squamous cell carcinoma specimens. Initially, to define tissue specific changes in expression compared to the reference RNA used, four oral mucosa specimen derived from healthy individuals were analyzed by CESH comparing them to human reference RNA chromosomal regions showing high mRNA expression were 1q36.2-p35, 1q31.2-q32.1, 2q23-q32.2, 5q31, 6q24, 10p13-p12, 10q22.3$\mathrm{q} 25,12 \mathrm{q} 13.12-\mathrm{q} 13.3,15 \mathrm{q} 22.1-\mathrm{q} 24,17 \mathrm{p} 12$ and 17q12-q21.1. Low mRNA expression was seen in the chromosomal regions 1q34 and 7q35. Consecutively, 28 OSCC specimens were analyzed by $\mathrm{CESH}$ in order to define chromosomal regions with altered average expression levels. Chromosomal regions exhibiting high mRNA expression in $>40 \%$ of cases examined are 1q22-q23, 1q31, 2q32-q34, 3q26.3-qter, 4q31.1-q32, 5q31-q34, 11q12-q13.2, 12q12-q14.1, 14q32, 17p12-pter, 17q11.2-q21.1, 18q12, 19q13.2-q13.3 and 22q13.1-q13.2. Low mRNA expression was found on chromosomal regions 7q35-q36, 8p22-p23, 16p12 and 16q23-q24. A summary of the results is presented in Fig. 1. A detailed summary of all chromosomal regions exhibiting changes in expression in $>40 \%$ of OSCC cases analysed is presented in Table I. On chromosomal regions 1q31, 2q32-q34, 5q31, $12 q 13,17 p 12$ and 17q12-q21.1, low mRNA expression was found in OSCC specimens and oral mucosa specimens as well, while decreased expression in both types of tissue was found on 7q35-qter. These findings most likely indicate tissue specific expression patterns. Conclusively, high mRNA expression of chromosomal regions 1q22-q23, 3q26.3-qter, 4q31.1-q32, 11q12-q13.2, 14q32, 18q12, 19q13.2-q13.3 and 22q13.1-q13.2 and low mRNA expression of chromosomal regions 8p22-p23, 16p12 and 16q23-q24 were considered to be specific for OSCC specimen.

\section{Discussion}

Genetic changes associated with malignant transformation and tumor progression critically affect the expression of key genes. Therefore, expression profiling approaches including microarray-based technologies are potential tools in cancer research. Global expression profiling has been successfully used for the molecular classification of tumors, identification of genetic markers, determination of the clinical behaviour of a tumor and prediction of oncogenic pathways (12-14). In this study, the method termed comparative expressed sequence hybridization (CESH) was used in order to search for new chromosome regions that may play a role in OSCC development. CESH is a relatively simple and rapid approach, which gives a genome-wide overview of relative expression patterns within tissues according to the chromosomal location $(2,15)$. The aim of the present study was to identify common chromosome regions, which are affected by differential mRNA expression profiles in OSCC specimens. Among others, such an aberrant high mRNA expression was 
Table I. Chromosome bands with high and low expression, respectively, comparing OSCC specimens to reference.

\begin{tabular}{|c|c|c|c|}
\hline $\begin{array}{l}\text { Chromosome bands } \\
\text { with high expression }\end{array}$ & $\begin{array}{c}\text { No. of cases } \\
(\%)\end{array}$ & $\begin{array}{l}\text { Chromosome bands } \\
\text { with high expression }\end{array}$ & $\begin{array}{c}\text { No. of cases } \\
(\%)\end{array}$ \\
\hline $1 q 22$ & $60.7 \quad(17)$ & $11 \mathrm{q} 12$ & $60.7 \quad(17)$ \\
\hline $1 q 23$ & $75 \quad(21)$ & $11 \mathrm{q} 13$ & $39.28(11)$ \\
\hline 1q31 & $57 \quad(16)$ & $12 q 12$ & $57 \quad(16)$ \\
\hline $2 \mathrm{q} 32.1-\mathrm{q} 32.2$ & $57 \quad(16)$ & $12 q 13$ & $60.7 \quad(17)$ \\
\hline $2 q 32.3-q 33$ & $71.4 \quad(20)$ & $14 q 32.1$ & $42.86(12)$ \\
\hline $2 q 24$ & $53.6 \quad(15)$ & $14 \mathrm{q} 32.2-\mathrm{q} 32.3$ & $53.57(15)$ \\
\hline $3 q 26.3-q 27$ & $75 \quad(21)$ & $17 \mathrm{p} 12$ & $67.85(19)$ \\
\hline $3 q 28$-qter & $71.4 \quad(20)$ & $17 \mathrm{p} 13$ & $67.85(19)$ \\
\hline $4 q 31.1$ & $60.7 \quad(17)$ & $17 \mathrm{p} 11.2$ & $46.42(13)$ \\
\hline $4 q 31.2-q 31.3$ & $75 \quad(21)$ & 17q11.2-q12 & $53.6 \quad(15)$ \\
\hline $4 q 32$ & $64 \quad(18)$ & $17 \mathrm{q} 21.1-\mathrm{q} 21.2$ & $46.42(13)$ \\
\hline $5 q 31$ & 67.85 (19) & $18 \mathrm{q} 12$ & $50 \quad(14)$ \\
\hline $5 q 32$ & $71.4 \quad(20)$ & $19 \mathrm{q} 13.1-\mathrm{q} 13.2$ & $50 \quad(14)$ \\
\hline $5 q 33$ & 60.7 & $22 q 13.1-q 13.2$ & $46.43(13)$ \\
\hline $\begin{array}{l}\text { Chromosome bands } \\
\text { with low expression }\end{array}$ & $\begin{array}{l}\text { No. of cases } \\
(\%)\end{array}$ & $\begin{array}{l}\text { Chromosome bands } \\
\text { with low expression }\end{array}$ & $\begin{array}{c}\text { No. of cases } \\
(\%)\end{array}$ \\
\hline $7 q 35$ & $75 \quad(21)$ & $16 \mathrm{p} 12$ & $53.57(15)$ \\
\hline \multirow[t]{2}{*}{$7 q 36$} & $78.75(22)$ & $16 q 23$ & $42.86(12)$ \\
\hline & & $16 q 24$ & $39.28(11)$ \\
\hline $8 \mathrm{p} 22$ & $57 \quad(16)$ & & \\
\hline $8 \mathrm{p} 23$ & $53.57(15)$ & & \\
\hline
\end{tabular}

frequently found for chromosomal bands 3q26.3-qter and 11q12-q13. Previous cCGH analyses suggested that DNA copy number gains of 3q26.3-qter and 11q12-q13 are driving genetic events in HNSCC progression, which are associated with adverse patient outcome (16). On the distal part of chromosome arm $3 \mathrm{q}$, several candidate proto-oncogenes have been identified in HNSCC and OSCC including PIK3CA (17), CCNLI (18) and ACK1 (19). For 11q12-q13, we described a distinct amplicon of recurrent DNA amplification in OSCC, which contains the proto-oncogenes CCNDI and CTTN (20). The $C C N D 1$ encoded protein cyclin D1 promotes the progression through the G1-S phase of the cell cycle by phosphorylation of the retinoblastoma protein. In addition, it is involved in the regulation of cellular growth, metabolism, and cellular differentiation (21). CTTN encodes cortactin, an actin-binding protein involved in cytoskeleton organization and cell adhesion structures. An increase of motility and invasiveness was described for HNSCC cell lines with CTTN amplification and cortactin overexpression, which decreased after selective cortactin knock-down (22). Retrospective studies found an association of amplification and adverse patient outcome in HNSCC (23). In this respect, the increase of transcriptional activity on chromosomal bands 3q26.3-qter and 11q12-q13 detected by CESH in our analysis is probably the result of distinct DNA copy number gains in these chromosome regions underlining the validity and reliability of the present experimental approach. Furthermore, it further points to the presence of candidate proto-oncogenes in 3q26.3-qter and 11q12-q13 in OSCC.

A novel critical chromosome region, which exhibited an increase in mRNA expression in OSCC, was chromosome 19q. In chromosomal comparative genomic (cCGH) analysis, the evaluation of chromosome 19 hybridization signals was considered to be critical due to a large amount of GC-rich DNA sequences (24). In recent array-CGH analyses, however, distinct regions of frequent DNA copy number gain were found on chromosome 19, including $19 \mathrm{q} 12$ and $19 \mathrm{q} 13.12$ (25). The proto-oncogene CCNE1 is located at $19 \mathrm{q} 12$, it is a member of the cyclin family, which has been shown to play an important role in G1/S transition of the cell cycle. Gene copy number gain of the CCNE1 locus and high cyclin E1 protein expression was found in about $25 \%$ of OSCC cases analyzed suggesting an activation of CCNE1 by gene copy number gain (25). According to our CESH data, the most frequent high mRNA expression is found on the distal chromosome bands 19q13.1-19q13.2. These findings are in concordance with a region of common DNA copy number gain in array-CGH analysis. While no candidate protooncogene is currently known in this chromosome region, the finding of recurrent high transcriptional activity of $19 q$ DNA 
sequences encourages further research activities to define the relevant proto-oncogenes.

Further chromosome regions of recurrent high mRNA expression detected in the present study are $14 \mathrm{q} 32$ and $22 q 13$, for which DNA copy number gain has been found in previous studies in OSCC (25-27). These findings underline the assumption that high mRNA expression levels detected by $\mathrm{CESH}$ reflect an increase in gene transcription, which is frequently the result of distinct DNA copy number gain.

A relative decrease of mRNA expression was found on 8p22-p23, which is in concordance with frequent DNA copy number loss on chromosome arm $8 \mathrm{p}$ in cCGH analysis in HNSCC $(16,28)$. For chromosome 18 , however, a relative increase of transcriptional activity was found in up to $50 \%$ of tumors analyzed in the present CESH analysis with the minimal overlapping region on $18 \mathrm{q} 12$. This was surprising, since monoallelic chromosome 18 deletions with several minimal overlapping regions have been previously described in OSCC and HNSCC, which were suggested to indicate a down-regulation of relevant tumor suppressor genes $(29,30)$. Furthermore, a loss of heterozygosity (LOH) affecting $18 \mathrm{q}$ was found associated with decreased patient survival (31). Subsequent studies identified candidate tumor suppressor genes inside these deleted regions including $D C C$ (32) and GALR1 (33), but failed to define a relevant impact of a loss of gene function on the clinical course of the disease. Due to the limited resolution of chromosomal CESH, the data do not allow to exactly delineate, which DNA sequences are responsible for the signal of increased expression in CESH experiments. However, the current findings do not provide evidence that segmental DNA copy number losses on chromosome 18 result in a reduced expression of tumor suppressor genes, which are critically involved in OSCC formation. To further investigate this question, the application of CESH to DNA arrays with high resolution might be helpful to define, which chromosome 18 regions and corresponding genes are actually subject of increased and decreased mRNA expression, respectively.

A major advantage of CESH analysis is the potential to define novel chromosome regions, which are subject of increased transcriptional activity, but were not yet investigated in detail, since no respective DNA copy number aberrations have been found. In the present analysis, a relative decrease of mRNA expression was detected on chromosome regions 16p12 and 16q23-q24, for which no DNA copy losses have been described in previous $\mathrm{CGH}$ analyses $(25,26,34)$. Candidate tumor suppressor genes in $16 \mathrm{p} 12$ are TNRC6A, which is involved in the regulation of mRNA silencing (35) and JMJD5, which may function in DNA mismatch repair (36). On $16 \mathrm{q} 23-\mathrm{q} 24$, the tumor suppressor gene WWOX is located, which was found inactivated in esophageal carcinoma (37). Further expression and functional studies are required to elucidate a possible role of these candidate genes in OSCC pathophysiology.

In conclusion, our study demonstrates that CESH is a reliable and potential technique for a genome-wide profiling of aberrant expressed chromosomal regions in OSCC. It allows the definition of consistent expression patterns characteristic for a tumor entity. Identification of targeted genes present in changed regions warrants further molecular investigation.

\section{Acknowledgements}

This study was supported in part by the National Genome Research Network (NGFN2/No. 01 GR 0417), the ManfredLautenschläger-Stiftung and the Medical Faculty of the University Heidelberg.

\section{References}

1. Jemal A, Siegel R, Ward E, Murray T, Xu J and Thun MJ: Cancer statistics, 2007. CA Cancer J Clin 57: 43-66, 2007.

2. Lu YJ, Williamson D, Clark J, et al: Comparative expressed sequence hybridization to chromosomes for tumor classification and identification of genomic regions of differential gene expression. Proc Natl Acad Sci USA 98: 9197-9202, 2001.

3. Gruszka-Westwood AM, Horsley SW, Martinez-Ramirez A, et al: Comparative expressed sequence hybridization studies of highhyperdiploid childhood acute lymphoblastic leukemia. Genes Chromosomes Cancer 41: 191-202, 2004.

4. Vanden Bempt I, Vanhentenrijk V, Drijkoningen M and De Wolf-Peeters C: Comparative expressed sequence hybridization reveals differential gene expression in morphological breast cancer subtypes. J Pathol 208: 486-494, 2006.

5. Vanhentenrijk V, De Wolf-Peeters C and Wlodarska I: Comparative expressed sequence hybridization studies of hairy cell leukemia show uniform expression profile and imprint of spleen signature. Blood 104: 250-255, 2004.

6. Vanhentenrijk V, Vanden Bempt I, Dierickx D, Verhoef G, Wlodarska I and De Wolf-Peeters C: Relationship between classic Hodgkin lymphoma and overlapping large cell lymphoma investigated by comparative expressed sequence hybridization expression profiling. J Pathol 210: 155-162, 2006.

7. Van den Boom J, Wolter M, Kuick R, et al: Characterization of gene expression profiles associated with glioma progression using oligonucleotide-based microarray analysis and real-time reverse transcription-polymerase chain reaction. Am J Pathol 163: 1033-1043, 2003.

8. Dries V, von Both I, Muller M, et al: Detection of hepatitis C virus in paraffin-embedded liver biopsies of patients negative for viral RNA in serum. Hepatology 29: 223-229, 1999.

9. Kirchhoff M, Gerdes T, Maahr J, et al: Deletions below 10 megabasepairs are detected in comparative genomic hybridization by standard reference intervals. Genes Chromosomes Cancer 25: 410-413, 1999.

10. Kirchhoff M, Gerdes T, Rose H, Maahr J, Ottesen AM and Lundsteen C: Detection of chromosomal gains and losses in comparative genomic hybridization analysis based on standard reference intervals. Cytometry 31: 163-173, 1998.

11. Pfaffl MW: A new mathematical model for relative quantification in real-time RT-PCR. Nucleic Acids Res 29: E45, 2001.

12. Golub TR, Slonim DK, Tamayo P, et al: Molecular classification of cancer: class discovery and class prediction by gene expression monitoring. Science 286: 531-537, 1999.

13. Shipp MA, Ross KN, Tamayo P, et al: Diffuse large B-cell lymphoma outcome prediction by gene-expression profiling and supervised machine learning. Nat Med 8: 68-74, 2002.

14. Alizadeh AA, Ross DT, Perou CM and van de Rijn M: Towards a novel classification of human malignancies based on gene expression patterns. J Pathol 195: 41-52, 2001.

15. Lu YJ, Williamson D, Wang R, et al: Expression profiling targeting chromosomes for tumor classification and prediction of clinical behavior. Genes Chromosomes Cancer 38: 207-214, 2003.

16. Bockmuhl U, Schluns K, Kuchler I, Petersen S and Petersen I: Genetic imbalances with impact on survival in head and neck cancer patients. Am J Pathol 157: 369-375, 2000.

17. Liu CJ, Lin SC, Chen YJ, Chang KM and Chang KW: Arraycomparative genomic hybridization to detect genomewide changes in microdissected primary and metastatic oral squamous cell carcinomas. Mol Carcinog 45: 721-731, 2006.

18. Sticht C, Hofele C, Flechtenmacher C, et al: Amplification of cyclin 11 is associated with lymph node metastases in head and neck squamous cell carcinoma (HNSCC). Br J Cancer 92: 770$774,2005$.

19. Van der Horst EH, Degenhardt YY, Strelow A, et al: Metastatic properties and genomic amplification of the tyrosine kinase gene ACK1. Proc Natl Acad Sci USA 102: 15901-15906, 2005. 
20. Freier K, Sticht C, Hofele C, et al: Recurrent coamplification of cytoskeleton-associated genes EMS1 and Shank2 with CCND1 in oral squamous cell carcinoma. Genes Chromosomes Cancer 45: 118-125, 2006

21. Fu M, Wang C, Li Z, Sakamaki T and Pestell RG: Minireview: cyclin D1: normal and abnormal functions. Endocrinology 145: 5439-5447, 2004.

22. Rothschild BL, Shim AH, Ammer AG, et al: Cortactin overexpression regulates actin-related protein $2 / 3$ complex activity, motility, and invasion in carcinomas with chromosome 11q13 amplification. Cancer Res 66: 8017-8025, 2006.

23. Rodrigo JP, Garcia-Carracedo D, Garcia LA, et al: Distinctive clinicopathological associations of amplification of the cortactin gene at $11 \mathrm{q} 13$ in head and neck squamous cell carcinomas. J Pathol 217: 516-523, 2009.

24. Du Manoir S, Schrock E, Bentz M, et al: Quantitative analysis of comparative genomic hybridization. Cytometry 19: 27-41, 1995.

25. Freier K, Knoepfle K, Flechtenmacher C, et al: Recurrent copy number gain of transcription factor SOX2 and corresponding high protein expression in oral squamous cell carcinoma. Genes Chromosomes Cancer 49: 9-16, 2010.

26. Weber RG, Scheer M, Born IA, et al: Recurrent chromosomal imbalances detected in biopsy material from oral premalignant and malignant lesions by combined tissue microdissection, universal DNA amplification and comparative genomic hybridization. Am J Pathol 153: 295-303, 1998.

27. Huang X, Gollin SM, Raja S and Godfrey TE: High-resolution mapping of the 11q13 amplicon and identification of a gene, TAOS1, that is amplified and overexpressed in oral cancer cells. Proc Natl Acad Sci USA 99: 11369-11374, 2002.

28. Gollin SM: Chromosomal alterations in squamous cell carcinomas of the head and neck: window to the biology of disease. Head Neck 23: 238-253, 2001.
29. Takebayashi S, Ogawa T, Jung KY, et al: Identification of new minimally lost regions on $18 \mathrm{q}$ in head and neck squamous cell carcinoma. Cancer Res 60: 3397-3403, 2000.

30. Blons H, Laccourreye O, Houllier AM, et al: Delineation and candidate gene mutation screening of the $18 \mathrm{q} 22$ minimal region of deletion in head and neck squamous cell carcinoma. Oncogene 21: 5016-5023, 2002.

31. Takebayashi S, Hickson A, Ogawa T, et al: Loss of chromosome arm $18 \mathrm{q}$ with tumor progression in head and neck squamous cancer. Genes Chromosomes Cancer 41: 145-154, 2004.

32. Carvalho AL, Chuang A, Jiang WW, et al: Deleted in colorectal cancer is a putative conditional tumor-suppressor gene inactivated by promoter hypermethylation in head and neck squamous cell carcinoma. Cancer Res 66: 9401-9407, 2006.

33. Misawa K, Ueda Y, Kanazawa T, et al: Epigenetic inactivation of galanin receptor 1 in head and neck cancer. Clin Cancer Res 14: 7604-7613, 2008.

34. Baldwin C, Garnis C, Zhang L, Rosin MP and Lam WL: Multiple microalterations detected at high frequency in oral cancer. Cancer Res 65: 7561-7567, 2005.

35. Schneider MD, Najand N, Chaker S, et al: Gawky is a component of cytoplasmic mRNA processing bodies required for early drosophila development. J Cell Biol 174: 349-358, 2006.

36. Suzuki T, Minehata K, Akagi K, Jenkins NA and Copeland NG: Tumor suppressor gene identification using retroviral insertional mutagenesis in BLM-deficient mice. EMBO J 25: 3422-3431, 2006.

37. Kuroki T, Trapasso F, Shiraishi T, et al: Genetic alterations of the tumor suppressor gene wwox in esophageal squamous cell carcinoma. Cancer Res 62: 2258-2260, 2002. 